\title{
FRACTIONAL ITERATION OF EXPONENTIALLY GROWING FUNGTIONS
}

\author{
G. SZEKERES \\ (received 24 July, 196I)

\section{Introduction}

The fractional iteration of $e^{x}$ and solutions of the functional equation (1)

$$
\phi(\phi(x))=e^{x}
$$

have frequently been discussed in literature. G. H. Hardy has shown (in [3], and in greater detail in [4]) that the asymptotic behaviour of the solutions of (1) cannot be expressed in terms of the logarithmico-exponential scale, although they are comparable with each member of the scale. ${ }^{1}$ Hence solutions of (1) provide a remarkably simple instance of functions whose manner of growth does not fit into the scale of $L$-functions but requires non-elementary orders of infinity for an accurate representation. This raises quite naturally the question whether there exists a most regularly growing solution of equation (1) which might serve as a prototype for this kind of growth. In a slightly more general context we may ask whether there exists a "best" family of fractional iterates $f_{\sigma}(x)$, satisfying

$$
f_{\sigma}\left(f_{\tau}(x)\right)=f_{\sigma+\tau}(x), \quad f_{1}(x)=e^{x}
$$

$H$. Kneser [5] has treated the problem from the point of view of analytic functions. The function $e^{z}$ has no real fixpoints (i.e. real roots of $e^{z}-z=0$ ) and this causes some difficulty in the analytical treatment of the problem. However by applying the method of Königs [6] in the neighbourhood of a complex fixpoint and subsequent conformal transformations Kneser succeeded in obtaining a real analytic solution of Abel's equation

$$
B\left(e^{x}\right)=B(x)+1
$$

from which he derived by a well known process the fractional iterates ${ }^{2}$

$$
f_{\sigma}(x)=B_{-1}(B(x)+\sigma) \text {. }
$$

In particular he obtained a real analytic solution of (1),

$$
\phi(x)=f_{\frac{1}{2}}(x)=B_{-1}\left(B(x)+\frac{1}{2}\right) .
$$

$1 f$ and $g$ are comparable if $f(x) / g(x)$ tends to a definite limit, finite or infinite. The logarithmico-exponential functions are briefly called $L$-functions.

1 For the sake of uniformity we denote by $B_{-1}(x)$ the inverse of $B(x)$. 
The solution of Kneser does not really solve the problem of "best" fractional iterates of $e^{x}$. Quite apart from practical difficulties involved in the calculation of Kneser's function on the real axis, there is no indication whatsoever that the function will grow more regularly to infinity than any other solution. There is certainly no uniqueness attached to the solution; in fact if $g(x)$ is a real analytic function with period 1 and $g^{\prime}(x)+1>0$ (e.g. $\left.g(x)=\frac{1}{2} \sin 2 \pi x\right)$ then

$$
B^{*}(x)=B(x)+g(B(x))
$$

is also an analytic Abel function of $e^{x} 3$ which in general yields a different solution of equation (1). Clearly the requirement of analyticity is not nearly sufficient to fix the solution uniquely.

The situation can best be illustrated with an analogy from the theory of the $\Gamma$-function. There the problem is to find the best possible solution of

$$
x \gamma(x)=\gamma(x+1), \quad \gamma(1)=1 .
$$

The equation has infinitely many real analytic solutions, and $\Gamma(x)$ happens to be one of them; but the requirement of analyticity plays no part whatsoever in the actual definition of the function. What distinguishes $\Gamma(x)$ uniquely among all solutions of (6) is logarithmic convexity, a genuinely real variable property (Bourbaki [2]). Alternatively we can characterize $\Gamma(x)$ as the only solution of (6) which is asymptotically equal to an $L$-function, viz. $(x / e)^{x}(2 \pi / x)^{ \pm}$. This analogy suggests at any rate that in search for a best solution of equation (1) it is far better to concentrate on the real variable and asymptotic properties than on the complex-analytic character of the solution.

In many cases of sub-exponential growth the most regular fractional iterates of a given $f(x)$ can be obtained by suitable asymptotic requirements. For instance if $f(x)=x+\omega(x)$ where $\omega(x) / x \rightarrow 0(x \rightarrow \infty)$ and $\omega^{\prime}(x)$ is of bounded variation then there exists a unique family of fractional iterates $f_{\sigma}(x)=x+\omega_{\sigma}(x)$ characterized by the condition

$$
\lim _{x \rightarrow \infty} \omega_{\sigma}(x) / \omega(x)=\sigma
$$

([8], [11]). Again if $f(x)=c x+\omega(x), c>1, \omega(x) / x \rightarrow 0$ and $\omega(x)$ is sufficiently well behaved at infinity then there is a unique family $f_{\sigma}(x)$ such that

$$
\lim _{x \rightarrow \infty} f_{\sigma}(x) / x=c^{\sigma}
$$

(See [10] for a detailed discussion).

We call an Abel function any continuous strictly increasing solution of Abel's equation.

- M. Kuczma [7] has discussed a similar situation recently. 
If we try to carry through this idea for the case of the exponential function we encounter the difficulty already mentioned earlier that there is no elementary asymptotics available for the fractional iterates. Nevertheless we can achieve a substantial simplification of the problem if we make use of an asymptotic classification of the iterates, by a method originally suggested by Paul Lévy [8].

Suppose that we have succeeded in locating an exponentially growing function $e(x)$ (not necessarily $e^{x}$ ) with a particularly well behaved Abel function $A(x)$ which we are prepared to accept as a standard of comparison for functions with a comparable manner of growth. Let $f(x)$ denote an arbitrary exponentially growing function which from the point of view of iteration is equivalent to $e(x)$, e.g. in the sense that

$$
e_{k-1}(x)<f_{k}(x)<e_{k+1}(x)
$$

for every $k \geqq 1 .^{s}$ Set

$$
a(x)=A^{\prime}(x) .
$$

Then under quite general conditions (which incidentally embrace all members of Hardy's scale) it can be shown that $f(x)$ possesses exactly one Abel function $B(x)$ (apart from an additive constant) such that its derivative $b(x)$ satisfies the asymptotic relation ${ }^{\circ}$

$$
\lim _{x \rightarrow \infty} b(x) / a(x)=1 .
$$

It seems therefore quite reasonable to declare $B(x)=\int^{x} b(t) d t$ as the best Abel function of $f(x)$ and the fractional iterates derived from $B(x)$ as the most acceptible solutions of the equations

$$
f_{\sigma}\left(f_{\tau}(x)\right)=f_{\sigma+\tau}(x), \quad f_{1}(x)=f(x) .
$$

Thus the whole problem is reduced to the selection of just one standard comparison function. We shall tackle the problem in $\S \mathbf{3}$ where it will be shown, by partly intuitive arguments, that a certain Abel function of $e(x)=e^{x}-1$ has the strongest claims to be nominated as the standard comparison function. Once the selection has been made, it will be possible to define (in $\S 4$ ) the best fractional iterates of almost any exponentially growing function, and in particular of all members of Hardy's scale.

The practical determination of the best iterates will be discussed in a subsequent joint paper with $\mathrm{K}$. W. Morris in which the necessary tables will also be supplied [9].

- $f<g$ means: $f(x) / g(x) \rightarrow 0$ when $x \rightarrow \infty$.

- Lévy has formulated his condition in terms of the Abel function itself (which he called the logarithm of iteration of $f(x)$ ), but it is far more convenient and unequivocal to state the condition in terms of the derivative. 


\section{The derived Abel function}

Throughout this and the following section, functions denoted by $f(x)$ or $g(x)$ will be supposed to be continuous, strictly monotone and differentiable for sufficiently large values of $x$. If in a statement the exact range of validity is not mentioned explicitly it will be assumed that it is valid for all sufficiently large values of the argument.

By an $A$ bel function of $f(x)$ we understand a strictly increasing continuous solution of

$$
B(f(x))=B(x)+1
$$

We assume that $B(x)$ is differentiable and call

$$
b(x)=B^{\prime}(x)
$$

the derived Abel function (d.A.f.) of $f(x)$. The derived Abel function seems to have a more fundamental significance for fractional iteration than the Abel function itself which has basically the character of an indefinite integral. For instance in all uniqueness theorems the Abel function will only be determined up to an additive constant and the fractional iterates are independent of the value of the constant.

We begin with reviewing some earlier results,

LEMMA 1. Let $f(x)$ be real and analytic for $x \geqq 0$ and suppose that $f(x)>x$, $f^{\prime}(x)>0$ for $x>0$;

$$
f(x)=x+a x^{2}+\cdots, \quad a>0 .
$$

Then $f(x)$ possesses a d.A.f. $b(x)$ with the asymptotic property

$$
\lim _{x \rightarrow 0+} x^{2} b(x)=1 / a \text {. }
$$

$b(x)$ is uniquely determined by this condition and is analytic for $x>0$. Moreover its higher derivatives satisfy the asymptotic relation

$$
\lim _{x \rightarrow 0+} x^{k+1} b^{(k-1)}(x)=(-1)^{k+1} k ! / a \quad(k \geqq 1) .
$$

Without the uniqueness statement the lemma is a special case of [10], Lemma 7. To prove uniqueness we note that

$$
b(f(x))=b(x) / f^{\prime}(x) .
$$

If $b^{*}(x)$ is a second d.A.f. and $b^{*}\left(x_{0}\right)=c b\left(x_{0}\right)$ for a given $x_{0}>0$ then by (6) and the corresponding equation $b^{*}(f(x))=b^{*}(x) / f^{\prime}(x)$ we get

$$
b^{*}\left(f\left(x_{0}\right)\right)=c b\left(f\left(x_{0}\right)\right)
$$

and generally for every $n>0$ 


$$
b^{*}\left(f_{-n}\left(x_{0}\right)\right)=c b\left(f_{-n}\left(x_{0}\right)\right) .
$$

But $f_{-1}(x)<x, \lim _{n \rightarrow \infty} f_{-n}\left(x_{0}\right)=0$, therefore by (4)

$$
\lim _{n \rightarrow \infty}\left(t_{-n}\left(x_{0}\right)\right)^{2} b^{*}\left(t_{-n}\left(x_{0}\right)\right)=c / a .
$$

Hence if $\lim _{x \rightarrow 0+} x^{2} b^{*}(x)$ exists at all, it must be equal to $c / a$, i.e. $c$ is a constant and $b^{*}(x)=c b(x)$ for every $x$. But

$$
\int_{x}^{f(x)} b^{*}(t) d t=\int_{x}^{f(x)} b(t) d t=1
$$

therefore $c=1$ and $b^{*}(x)=b(x)$ for every $x$. We conclude that $b(x)$ is the only d.A.f. of $f(x)$ for which $\lim _{x \rightarrow 0+} x^{2} b(x)$ exists.

Following the terminology introduced in [10] we shall call $B(x)=\int^{x} b(t) d t$ the regular Abel function of $f(x), b(x)$ the regular d.A.f. and the corresponding iterates

$$
f_{\sigma}(x)=B_{-1}(B(x)+\sigma)
$$

the regular iterates of $f(x)$ (relative to $x=0$ ). The regular iterates are analytic for $x>0^{7}$ and they have an asymptotic development of the form

$$
f_{\sigma}(x)=\sum_{n=1}^{\infty} a_{n}{ }^{(\sigma)} x^{n} \quad(x \rightarrow 0+)
$$

where $a_{1}{ }^{(\sigma)}=1, a_{2}{ }^{(\sigma)}=\sigma a$ and $a_{n}{ }^{(\sigma)}$ for $n>2$ is obtained by comparing coefficients in the formal identity

$$
f_{\sigma}\left(f(x)=f\left(f_{\sigma}(x)\right)\right. \text {. }
$$

LemMa 2. If $b(x)$ is a d.A.f. of $f(x)$ and $c$ is any positive number then $c b(c x)$ is ad.A.f. of $c^{-1} f(c x)$, and generally $\phi^{\prime}(x) b(\phi(x))$ is ad.A.f. of $\phi_{-1}(f(\phi(x)))$. If moreover $f(x)$ satisfies the conditions of Lemma 1 and $b(x)$ is the regular d.A.f. (relative to $x=0$ ) of $f(x)$ then $c b(c x)$ is the regular d.A.f. of $c^{-1} f(c x)$.

Proof follows immediately from the definition of regularity and of the d.A.f.

LEMMA 3. Let $\lambda(x)$ be monotone increasing and twice differentiable, $\lambda^{\prime}(x)>0$ and suppose that $\mu(x)=1 / \lambda^{\prime}(x)$ satisfies the conditions

$$
\begin{aligned}
& \lim _{x \rightarrow \infty} \mu(x)=q<1, \\
& x\left|\mu^{\prime}(x)\right|<K<\infty .
\end{aligned}
$$

Let $\beta(x)$ be a d.A.f. of $\lambda(x)$ such that $\beta^{\prime}(x)$ exists. Then for given $c>0$

7 Not necessarily at $x=0$ itself, as shown by the example of $f(x)=e^{x}-1$ (I. N. Baker [1], Satz 17). 


$$
\lim _{x \rightarrow \infty} \beta(x+c) / \beta(x)=1 \text {. }
$$

Proof: The statement follows if we can prove that

$$
\beta^{\prime}(x)=o(\beta(x)) \quad(x \rightarrow \infty) .
$$

Now by definition

$$
\lambda^{\prime}(x) \beta(\lambda(x))=\beta(x), \quad \lambda^{\prime \prime}(x) \beta(\lambda(x))+\left(\lambda^{\prime}(x)\right)^{2} \beta^{\prime}(\lambda(x))=\beta^{\prime}(x)
$$

hence

$$
\frac{\beta^{\prime}(\lambda(x))}{\beta(\lambda(x))}=\mu(x) \frac{\beta^{\prime}(x)}{\beta(x)}+\mu^{\prime}(x) .
$$

Write $q_{1}=\frac{1}{2}(q+1)<1$, then by (8)

$$
\mu(x)<q_{1}, \quad \lambda(x)>x / q_{1}, \quad \lambda_{n}(x)>x / q_{1}{ }^{n}
$$

for large $x$ and we find by induction from (12), (13) and (9)

$$
\frac{\beta^{\prime}\left(\lambda_{n}(x)\right)}{\beta\left(\lambda_{n}(x)\right)}<q_{1}{ }^{n} \frac{\beta^{\prime}(x)}{\beta(x)}+n K q_{1}{ }^{n-1} / x,
$$

provided that $x$ is sufficiently large. This proves (11).

By combining Lemma 3 with Lemma 2 we find:

LEMMA 4. Let $f(x)=\exp (\lambda(\log x))$ where $\mu(x)=1 / \lambda^{\prime}(x)$ satisfies the conditions (8) and (9). Let $b(x)$ be a d.A.f. of $f(x)$ such that $b^{\prime}(x)$ exists. Then for given $c>0$,

$$
\lim _{\infty \rightarrow \infty} \frac{c b(c x)}{b(x)}=1 \text {. }
$$

It is easy to verify that $g(x)=1 / f^{\prime}(x)$ satisfies the conditions

$$
\lim _{x \rightarrow \infty} g(x)=0, \lim _{x \rightarrow \infty} g^{\prime}(x)=0
$$

so that Lemma 3 is applicable to $f(x)$ and we find:

THEOREM 1. Let $f(x)$ be as in Lemma 4 and $b(x)$ a differentiable d.A.f. of $f(x)$. Let $c>0$ and $d$ any real number. Then $b^{*}(x)=c b(c x+d)$ is a d.A.f. of $f^{*}(x)=f(c x+d)$ and

$$
\lim _{a \rightarrow \infty} b^{*}(x) / b(x)=1
$$

\section{Regular iteration of $e^{x}-1$}

It follows from Lemma 1, applied to the particular case of 


$$
e(x)=e^{x}-1,
$$

that $e(x)$ has a unique d.A.f. $a(x)$,

$$
a(e(x))=e^{-x} a(x),
$$

such that

$$
\lim _{x \rightarrow 0+}(-1)^{k+1} x^{k+1} a^{(k-1)}(x)=2 k ! \quad(k>1) .
$$

The corresponding fractional iterates will be denoted by $e_{\sigma}(x)$.

The asymptotic conditions (3) refer of course to the behaviour of $a(x)$ in the neighbourhood of 0 and express the fact that

$$
A(x)=\int{ }^{x} a(t) d t
$$

is the best behaved Abel function of $e(x)$ near $x=0$. It was Paul Lévy [8] who first suggested that this same Abel function might also be best behaved at infinity. Lévy has based his suggestion on a general assumption which, as we shall see later, is wholly unjustified. Nevertheless in the particular case of $e(x)$ Lévy's claim appears to be correct and it is the main purpose of our present discussion to give more substance to this hypothesis.

We call $f(x)$ totally monotonic at $x_{0}$ if it has derivatives of any order and

$$
(-1)^{k+1} f^{(k)}\left(x_{0}\right)>0 \text { for every } k>0 .
$$

Denote by $\boldsymbol{M}$ the class of real functions $F(x)$ defined for $x>0$ and totally monotonic at every $x>0 ; \log x$ is a typical representative of $M$. The following property of $\boldsymbol{M}$ is trivial:

LEMMA 5. Let $F(x) \in M$ and

$$
G(x)=a F(b x+c)+d
$$

where $a>0, b>0, c>0$ and $d$ is any real constant. Then also $G(x) \in M$. We shall prove now:

THEOREM 2. Let $A(x)$ denote the Abel function (4) of $e(x)$, determined by the asymptotic property (3). Then $A(x) \in M$ and $A(x)$ is the only Abel function of $e(x)$ with this property.

Since total monotonity usually indicates a strong degree of regularity of growth, Theorem 2 makes it at least very plausible that $a(x)$ is a good choice for the standard d.A.f.

Theorem 2 is a consequence of

THEOREM 3. Let $f(x)$ be as in Lemma 1 and suppose that $F(x)=f_{-1}(x) \in M$. Let $b(x)$ be the regular d.A.f. of $f(x)$ (relative to $x=0$ ); then also $B(x)=$ $\int^{x} b(t) d t$ is in $M$. 
If $b^{*}(x)$ is a second d.A.f. of $f(x)$, distinct from $b(x)$, then $B^{*}(x)=\int{ }^{x} b^{*}(t) d t$ is not in $M$.

Clearly Theorem 2 follows from Theorem 3 by setting $F(x)=\log (1+x)$. Incidentally the first half of the theorem is also valid for analytic functions of the form

$$
f(x)=c x+\cdots, \quad c>1,
$$

but not the uniqueness statement.

Proof of Theorem 3. Suppose that $f(x)$ is as in Lemma 1. By definition of $b(x)$ we have

$$
F^{\prime}(x) \cdot b(F(x))=b(x)
$$

and this with $(2.4)^{8}$ gives immediately $b(x)>0$ for $x>0$. Note that $F(x)<x$ so that $\lim _{n \rightarrow \infty} F_{n}(x)=0$ for every given $x>0$.

Differentiating (7) we get

$$
F^{\prime \prime}(x) \cdot b(F(x))+\left(F^{\prime}(x)\right)^{2} \cdot b^{\prime}(F(x))=b^{\prime}(x) .
$$

The first term on the left is negative because $F^{\prime \prime}(x)<0$, therefore $b^{\prime}(x) \geqq 0$ implies $b^{\prime}(F(x))>0$ and hence $b^{\prime}\left(F_{n}(x)\right)>0$ for every $n>0$. This contradicts (2.5) with $k=2$. Hence $b^{\prime}(x)<0$ for every $x>0$. The proof of $(-1)^{k} b^{(k)}(x)>0$ for $k>2$ is obtained similarly, by repeated differentiation of (8) and induction on $k$.

Suppose now that $b^{*}(x)$ is an arbitrary continuous d.A.f. of $f(x)$ so that $F^{\prime}(x) \cdot b^{*}(F(x))=b^{*}(x)$. It follows fom (7) that

$$
b^{*}(F(x)) / b(F(x))=b^{*}(x) / b(x) \text { from every } x>0 .
$$

Since

$$
\int_{F(x)}^{*} b(t) d t=\int_{F(x)}^{x} b^{*}(t) d t=1
$$

and $b(x), b^{*}(x)$ are not identical, we must have

$$
b^{*}\left(x_{1}\right)=(1+\delta) b\left(x_{1}\right), \quad \delta>0
$$

for some $x_{1}>0$ and

$$
b^{*}\left(x_{2}\right)<b\left(x_{2}\right)
$$

for some $x_{2}, x_{1}<x_{2}<f\left(x_{1}\right)$. From (9) we conclude that

$$
\begin{gathered}
b^{*}\left(F_{n}\left(x_{1}\right)\right)=(1+\delta) b\left(F_{n}\left(x_{1}\right)\right), \\
b^{*}\left(F_{n}\left(x_{2}\right)\right)<b\left(F_{n}\left(x_{2}\right)\right)
\end{gathered}
$$

(2.4) refers to formula (4) of $\S 2$. 
for every $n \geqq 0$.

Let $n$ be large and write

$$
\xi_{1}=F_{n}\left(x_{1}\right), \quad \xi_{2}=F_{n+1}\left(x_{2}\right), \quad \xi_{3}=F_{n}\left(x_{2}\right)
$$

so that $\xi_{2}=F\left(\xi_{3}\right)<\xi_{1}<\xi_{3}$. Then by (12), (13),

$$
b^{*}\left(\xi_{1}\right)=(1+\delta) b\left(\xi_{1}\right), \quad b^{*}\left(\xi_{2}\right)<b\left(\xi_{2}\right), \quad b^{*}\left(\xi_{3}\right)<b\left(\xi_{3}\right)
$$

and

$$
\begin{aligned}
& b^{*}\left(\xi_{1}\right)-b^{*}\left(\xi_{2}\right)>\delta b\left(\xi_{1}\right)-\left(b\left(\xi_{2}\right)-b\left(\xi_{1}\right)\right), \\
& b^{*}\left(\xi_{1}\right)-b^{*}\left(\xi_{3}\right)>(1+\delta) b\left(\xi_{1}\right)-b\left(\xi_{3}\right)>0
\end{aligned}
$$

since $b\left(\xi_{1}\right)>b\left(\xi_{3}\right)$. But if $\xi_{1}$ is sufficiently small then by $(2.5)$

and

$$
0<\frac{b\left(\xi_{2}\right)-b\left(\xi_{1}\right)}{\xi_{1}-\xi_{2}}<-b^{\prime}\left(\xi_{2}\right)<3 / a \xi_{2}^{3},
$$

$$
b\left(\xi_{2}\right)-b\left(\xi_{1}\right)<3\left(\xi_{1}-\xi_{2}\right) / a \xi_{2}^{3}<4 / \xi_{2}<\delta / 2 a \xi_{1}^{2}<\delta b\left(\xi_{1}\right)
$$

since $\xi_{1}-\xi_{2}<\xi_{3}-\xi_{2}=f\left(\xi_{2}\right)-\xi_{2}<\frac{4}{3} a \xi_{2}^{2}$ for small $\xi_{2}$, by (2.3). Hence by (14) and (15), $b^{*}\left(\xi_{1}\right)-b^{*}\left(\xi_{2}\right)>0, b^{*}\left(\xi_{1}\right)-b^{*}\left(\xi_{3}\right)>0$ and we conclude that $b^{* \prime}(x)$ takes both positive and negative values in the interval $F_{n+1}\left(x_{2}\right) \leqq x \leqq F_{n}\left(x_{2}\right)$, provided that $n$ is sufficiently large. This shows that $b^{*}(x)$ cannot be totally monotonic (not even concave) near the origin, and the theorem is proved.

Let us agree for the moment that on the evidence of Theorem 2, we are entitled to declare $a(x)$ to be the best Abel function of $e(x)$. Since $a(x)$ tends to infinity more slowly than any finite iterate of $\log x$ (and its inverse grows more rapidly than any iteration of $e^{x}$ ), we have indeed succeeded in extending Hardy's scale by a perfectly reasonable new order of infinity. There is nothing to prevent us from going a step further and apply Theorem 3 to the function

$$
F(x)=F(x, t)=\frac{A(x+t)-A(t)}{A^{\prime}(t)}=\frac{1}{a(t)} \int_{t}^{x+t} a(u) d u
$$

where $t$ is a fixed positive number. By Lemma 5 and Theorem 2, $F(x)$ is analytic for $x \geqq 0, F^{\prime}(0)=1$ and $F(x) \in M$. Therefore Theorem 3 is applicable and we obtain a uniquely determined totally monotonic Abel function $B(x)=B(x, t)$ of $F(x)$, which by the same taken as above can be regarded as the most acceptible Abel fuction of $F(x)$. But then, by the asymptotic argument presented at the end of $\S 1$, we would expect

$$
\lim _{x \rightarrow \infty} b\left(x, t_{1}\right) / b\left(x, t_{2}\right)=1
$$


to be true for the d.A.f.'s corresponding to arbitrary pairs of positive numbers $t_{1}, t_{2}{ }^{\circ}$

Now Lévy assumed in [8] that condition (17) is indeed satisfied by the (relative to 0 ) regular d.A.f.'s of any pair of functions $g\left(x, t_{1}\right), g\left(x, t_{2}\right)$ which have been obtained from a given $f(x)$ by

$$
g(x)=g(x, t)=\frac{f(x+t)-f(t)}{f^{\prime}(t)},
$$

provided that $f(x)$ grows to infinity with sufficient regularity. It was precisely this assumption which formed the basis of his proposed definition of regular growth. Lévy himself left the theory at a fairly intuitive stage with the remark that it would be quite difficult to pursue it much further. At any rate it seems that the theory has never been put to a serious test. In the remaining part of this section we intend to show, by a semi-heuristic argument, that Lévy's condition is almost certainly not fulfilled by any $f(x)$ which grows at least exponentially to infinity and which is essentially different from $e^{x}-1$. The point of the argument is that for such functions the iterates which behave well near 0 do not behave in the best possible manner at infinity, not even in cases of total monotonity of the Abel function. However in the course of the discussion the exceptional position of $e^{x}-1$ will become quite evident and the acceptance of $a(x)$ as the standard representative of the new order of infinity seems to be wholly justified. Any further extension of Hardy's scale and in particular the selection of a best representative among the $b(x, t)$ would probably require an essentially new principle which is certainly beyond the scope of the present paper.

It is obviously not a great restriction of generality if we confine ourselves to functions which satisfy the conditions of Lemma 4 and Theorem 1 . If for such a function Lévy's condition (17) is satisfied then by Theorem 1 it also holds for $g^{*}(x)=c^{-1} g(c x)$. Hence we can normalize $g^{*}(x)$ so that $g^{* \prime \prime}(0)=1$, and we can state Levy's condition for regularity as follows:

Given $f(x)$ consider

$$
g(x)=g(x, t)=[f(c x+t)-f(t)] / c f^{\prime}(t)
$$

where

$$
c=f^{\prime}(t) / f^{\prime \prime}(t) .
$$

Let $b(x, t)$ denote the (relative to 0 ) regular d.A.f. of ${ }^{2} g(x)$ characterized by the property

$$
\lim _{x \rightarrow 0+} x^{2} b(x, t)=2 .
$$

- The fact that $F\left(x, t_{2}\right)$ has a d.A.f. with property (17) follows from Theorem 1. 
$f(x)$ is said to be regularly growing if condition (17) holds for any pair of positive numbers $t_{1}, t_{2}$.

To demonstrate the inadequacy of Lévy's definition we prove a general result concerning the functions $g(x, t)$ which is interesting for its own sake.

THEOREM 4. Let $f(x)=\exp \lambda(\log x)$ be monotone increasing and twice differentiable,

$$
\lim _{x \rightarrow \infty} \mu(x)=0
$$

$$
\lim _{x \rightarrow \infty} \mu^{\prime}(x)=0
$$

where $\mu(x)=1 / \lambda^{\prime}(x)$.

Set

$$
g(x)=g(x, t)=[f(c x+t)-f(t)] / c f^{\prime}(t)
$$

where

$$
c=f^{\prime}(t) / f^{\prime \prime}(t)
$$

Then for every fixed $x>0$

$$
\lim _{t \rightarrow \infty} g(x, t)=e^{x}-1 .
$$

The theorem states essentially that if the origin is displaced to the point $(t, f(t))$ and the graph of the function is expanded linearly in the two coordinate directions until the first and second derivatives both become 1 then in a certain right neighbourhood of 0 the function will become almost identical with $e^{x}-1$, irrespective of what the function $f(x)$ was. It will follow from the proof that the length of the interval $(0, \xi)$ on which the phenomenon occurs is of order $\lambda^{\prime}(\log t)$.

Proof: We have (for fixed $t$ )

$$
\begin{aligned}
g(x) & =\frac{t}{c \lambda^{\prime}(\log t)}[\exp \{\lambda(\log (c x+t))-\lambda(\log t)\}-1], \\
c & =t\left(\lambda^{\prime}(\log t)-1+\lambda^{\prime \prime}(\log t) / \lambda^{\prime}(\log t)\right)^{-1}
\end{aligned}
$$

hence writing $u=\log t$,

$$
g(x)=\eta(u)\left[\exp \left\{\lambda\left(u+\log \left(1+\frac{x}{\eta(u) \lambda^{\prime}(u)}\right)\right)-\lambda(u)\right\}-1\right]
$$

where

$$
\eta(u)=1-\mu(u)-\mu^{\prime}(u) .
$$


But

$$
\lim _{u \rightarrow \infty} \eta(u)=1
$$

by (22) and (23) and therefore

$$
\begin{aligned}
\lambda\left(u+\log \left(1+\frac{x}{\eta(u) \lambda^{\prime}(u)}\right)\right) & =\lambda(u)+x \frac{\mu(u)(1+o(1))}{\mu(u+\theta \mu(u))} \\
& =\lambda(u)+x(1+o(1))
\end{aligned}
$$

for $0<x \leqq \xi$, where $\theta$ is bounded and $o(1)$ refers to $u \rightarrow \infty$. Hence by (25) and (26), $\left(g(x) \rightarrow e^{x}-1\right.$ when $u \rightarrow \infty$.

Qualitatively we can describe the behaviour of $g(x, t)$ as follows: For small $x$ the function is almost identical with $e^{x}-1$ until $x$ reaches the order of magnitude $\lambda^{\prime}(\log t)$. Then follows a comparatively short transition region (short in terms of the index (or logarithm) of iteration) in which it briskly changes its character of growth to something approaching the behaviour of $f(x)$. This abrupt change in behaviour is reflected in a corresponding change of character of the derived Abel function: the two Abel functions which are best behaved to the left and to the right of the critical region do not pass into each other when the region is traversed.

Let us illustrate the situation by the example of $f(x)=\exp \left(e^{x}\right)$. We have

$$
g(x, t)=\left(1+e^{-t}\right)\left[\exp \left\{e^{t}\left(\exp \frac{x}{e^{t}+1}-1\right)\right\}-1\right]
$$

and the critical region is in the neighbourhood of $x_{0}=t e^{t}$. Let $a^{*}(x)$ denote the (relative to 0 ) regular d.A.f. of $g(x)$ and consider $x_{1}=e_{\sigma_{1}}(t), x_{2}=e_{\sigma_{2}}(t)$, where $0<\sigma_{1}<\sigma_{2}<1$. In terms of the best Abel function to the left of $e(t)$ the iteration interval of $\left(x_{1}, x_{2}\right)$ is approximately of length $\sigma_{2}-\sigma_{1}$. But the interval $\left(y_{1}, y_{2}\right)=\left(g\left(x_{1}\right), g\left(x_{2}\right)\right)$ is not very different from $\left(e_{1+\sigma_{1}}(t), e_{1+\sigma_{2}}(t)\right)$ and this is approximately of iteration length $\frac{1}{2}\left(\sigma_{2}-\sigma_{1}\right)$ in terms of the behaviour of $\exp \left(e^{x}\right)$ (hence of $g(x)$ ) in the interval $\left(y_{1}, y_{2}\right)$. Therefore the "best" d.A.f. $b(x)=b(x, t)$ at $y_{1} \leqq x \leqq y_{2}$ is approximately equal to $\frac{1}{2} a^{*}(x)$, and the same is true for any $x$ with $y_{1} \leqq g_{-n}(x) \leqq y_{2}$ where $n$ is a non-negative integer.

On the other hand, since

$$
\int_{x}^{o(x)} a^{*}(t) d t=\int_{x}^{o(x)} b(t) d t=1,
$$

$b(x) / a^{*}(x)$ is bound to take large values (considerably greater than 1 ) if $g_{-n}(x)$ happens to be in the neighbourhood of $x_{0}$. Hence the value of $b(x, t) / a^{*}(x)$ will depend very sensitively on the relative positions of $x$ and $t$, and for given $t_{1} \neq t_{2}$ the value of $b\left(x, t_{1}\right) / b\left(x, t_{2}\right)$ will fluctuate between small 
and large values as $x$ goes to infinity. This heuristic argument can be made quite precise (at least in the case of exp $\left(e_{x}\right)$ ), but our purpose here was merely to show that Lévy's condition is extremely unlikely to hold for fast growing functions.

If the growth of $f(x)$ is not very different from the growth of $e^{x}$ then of course there is no break in the behaviour of the d.A.f. at the critical region and in the limiting case of $f(x)=e^{x}-1$ Lévy's condition is trivially fulfilled since each $g(x, t)$ is equal to $e(x)=e^{x}-1$ and $b(x, t)=a(x)$ does not depend on $t$ at all. Therefore the assumption that $a(x)$ is the best behaved d.A.f., not only at 0 but also at infinity, seems to be very well substantiated.

\section{Regular iteration of $L$-functions}

As in the previous section, $e(x)$ denotes $e^{x}-1$ and $a(x)$ is the regular d.A.f. of $e(x), A(x)=\int_{1}^{x} a(t) d t$ the regular Abel function of $e(x)$, normalized so that $A(1)=0$. Our purpose is to show that if $a(x)$ is added to Hardy's scale then the scale becomes effective for the fractional iteration of almost any function which occurs in practice; in particular we can determine unique best iterates for every $L$-function. We recall that $L$-functions are members of the smallest set $\boldsymbol{H}$ such that

(i) $\boldsymbol{H}$ contains the constant functions $f(x)=c$ and the identity function $f(x)=x$.

(ii) $\boldsymbol{H}$ is closed under the rational operations and the application of $\exp ($ ) and $\log 11$.

To avoid awkward brackets we shall use the notation $f \circ g(x)$ for $f(g(x))$.

Definition. We say that $f(x)$ is regular with respect to iteration if it has the form

$$
f(x)=A_{-1} \circ g \circ A(x)
$$

where

$$
g(x)=x+\omega(x), \lim _{x \rightarrow \infty} \omega(x) / x=0
$$

and $g(x)$ has a family of iterates

$$
g_{\sigma}(x)=x+\omega_{\sigma}(x)
$$

with

$$
\lim _{x \rightarrow \infty} \omega_{\sigma}(x) / \omega(x)=\sigma .
$$

For instance $f(x)$ is certainly regular if it has the form (1) and (2) and $\omega^{\prime}(x)$ is of bounded variation. 
The (relative to $\infty$ ) regular iterates $f_{\sigma}(x)$ of $f(x)$ are given by

$$
f_{\sigma}(x)=A_{-1} \circ g_{\sigma} \circ A(x)
$$

and they are uniquely determined by condition (4). We shall find that all members of Hardy's scale are regular by this definition.

First we confirm that the above definition of regularity is in no conflict with earlier definitions in [10].

TheOREM 5. Suppose that $g(x)$ is as in (2) and that it has a family of iterates $g_{\sigma}(x)$ with property (3). Let

$$
f(x)=e_{r} \circ g \circ e_{-r}(x)
$$

where $r$ is a non-negative integer. Then $f(x)$ is regular with respect to iteration and its regular iterates are given by

$$
f_{\sigma}(x)=e_{r} \circ g_{\sigma} \circ e_{\rightarrow r}(x) .
$$

In particular, the regular iterates of $g(x)$ itself are given by $g_{\sigma}(x)$.

Proof. Define $f_{\sigma}(x)$ by (7). Then

$$
\begin{aligned}
A \circ f_{\sigma} \circ A_{-1}(x) & =A \circ e_{r} \circ g_{\sigma} \circ e_{-\rightarrow} \circ A_{-1}(x) \\
& =A \circ g_{\sigma} \circ A_{-1}(x-r)+r \\
& =A\left(A_{-1}(x-r)+\omega_{\sigma} \circ A_{-1}(x-r)\right)+r \\
& =x+\omega_{\sigma} \circ A_{-1}(x-r) \cdot A^{\prime}\left(A_{-1}(x-r)+\theta(x) \cdot \omega_{\sigma} \circ A_{-1}\right. \\
& \quad(x-r)), \quad 0<\theta(x)<1 .
\end{aligned}
$$

But

$$
\lim _{x \rightarrow \infty} \omega_{\sigma} \circ A_{-1}(x-r) / \omega \circ A_{-1}(x-r)=\sigma
$$

by (4) and

$$
\lim _{x \rightarrow \infty} A^{\prime}\left(A_{-1}(x-r)+\theta(x) \omega_{\sigma} \circ A_{-1}(x-r)\right) / A^{\prime}\left(A_{-1}(x-r)\right)=1
$$

by the following lemma:

Lemma 6. Suppose that

Then

$$
\lim _{x \rightarrow \infty} \eta(x) / x=0
$$

$$
a(x+\eta(x)) / a(x)=1+0\left(\frac{\eta(x)}{x}\right)
$$

Proof. From (3.2)

$$
\frac{a^{\prime}(e(x))}{a(e(x))}=e^{-\infty}\left(\frac{a^{\prime}(x)}{a(x)}-1\right)
$$


whence we get

$$
0<\frac{a^{\prime}(x)}{a(x)}<\frac{2}{x} \text { for } x>x_{0}
$$

and

$$
\begin{aligned}
a(x+\eta(x)) & =a(x)+\theta(x) \eta(x) a^{\prime}(x)=a(x)-\frac{2 \theta_{1}(x)}{x} \eta(x) a(x) \text { if } \eta(x)>0, \\
a(x+\eta(x)) & =a(x)+\theta(x) \eta(x) a^{\prime}(x+\eta(x)) \\
& =a(x)-\frac{2 \theta_{1}(x)}{x+\eta(x)} \eta(x) a(x+\eta(x)) \text { if } \eta(x)<0
\end{aligned}
$$

where $0<\theta(x)<1,0<\theta_{1}(x)<1$.

THEOREM 6. Suppose that $f(x)$ has a d.A.f. $b(x)$ such that for some $\gamma>0$,

$$
\lim _{x \rightarrow \infty} a(x) / b(x)=\gamma .
$$

Then $f(x)$ is regular with respect to iteration and its regular iterates are given by

$$
f_{\sigma}(x)=B_{-1}(B(x)+\sigma), \quad B(x)=\int^{x} b(t) d t .
$$

Proof. Define $f_{\sigma}(x)$ by (11) and consider

$$
g_{\sigma}(x)=A \circ f_{\sigma} \circ A_{-1}(x)=\phi\left(\phi_{-1}(x)+\sigma\right)
$$

where

We have

$$
\phi(x)=A \circ B_{-1}(x)
$$

$$
\phi^{\prime} \circ B(x)=A^{\prime}(x) / B^{\prime}(x)=a(x) / b(x) \rightarrow \gamma \quad(x \rightarrow \infty) .
$$

But (12) gives

$$
g_{\sigma}(x)=x+\sigma \phi^{\prime}\left(\phi_{-1}(x)+\sigma \theta(x)\right)=x+\omega_{\sigma}(x), 0<\theta(x)<1,
$$

where by (13)

$$
\lim _{x \rightarrow \infty} \omega_{\sigma}(x)=\sigma \gamma
$$

and so (4) is satisfied.

THEOREM 7. Suppose that

$$
f(x)=e_{r}(x+\omega(x))
$$

where $r$ is a positive integer and

$$
\begin{gathered}
|\omega(x)| \leqq K, \\
\omega^{\prime}(x)=0(1 / x) \quad(x \rightarrow \infty) .
\end{gathered}
$$


Then $f(x)$ has a d.A.f. $b(x)$ such that

$$
\lim _{x \rightarrow \infty} a(x) / b(x)=r .
$$

$b(x)$ is continuous and is uniquely determined by condition (17).

In particular the theorem is true if $\omega(x)$ is bounded and ultimately monotonic. The theorem enables us to determine the asymptotically best behaved Abel function and fractional iterates of any $f(x)$ of the form (1) and for instance to determine the best solution of equation (1.1). Because of the continuity of $b(x)$, these iterates have a continuous first derivative, provided that $f^{\prime}(x)$ itself is continuous.

Proof. Suppose first that a d.A.f. with property (17) does actually exist. For fixed $x$

$$
f^{\prime}(x) \cdot b(f(x))=b(x)
$$

hence by repeated application

$$
b \circ f_{n}(x)=b(x) / \prod_{i=0}^{n-1} f^{\prime} \circ f_{i}(x) .
$$

By (17)

$$
b \circ f_{n}(x) / a \circ f_{n}(x)=b(x) / a \circ f_{n}(x) \cdot \prod_{i=0}^{n-1} f^{\prime} \circ f_{i}(x) \rightarrow 1 / r(n \rightarrow \infty)
$$

so that

$$
b(x)=\lim _{x \rightarrow \infty} \frac{1}{r} a \circ f_{n}(x) \cdot \prod_{i=0}^{n-1} f^{\prime} \circ f_{i}(x) .
$$

Therefore if $b(x)$ exists at all, it is given by formula (18) and so it is uniquely determined.

Conversely if $b(x)$ is defined by (18) then

$$
b \circ f(x)=\lim _{n \rightarrow \infty} \frac{1}{r} a \circ f_{n+1}(x) \cdot \prod_{i=0}^{n-1} f^{\prime} \circ f_{i+1}(x)=b(x) / f^{\prime}(x)
$$

so that $b(x)$ is (apart from a constant factor) the derivative of an Abel function of $f(x)$.

To prove the convergence of (18), denote by $a_{n}$ the expression on the right hand side of (18). We have

$$
a_{n+1} / a_{n}=\left(a \circ f_{n+1}(x) / a \circ f_{n}(x)\right) \cdot f^{\prime} \circ f_{n}(x)=a \circ f(y) \cdot f^{\prime}(y) / a(y)
$$

where $y=f_{n}(x) \rightarrow \infty$ when $n \rightarrow \infty$. But

$$
\begin{aligned}
& f(y)=e_{r}(y+\omega(y)), f^{\prime}(y)=\left(1+\omega^{\prime}(y)\right) e_{r}^{\prime}(y+\omega(y)), \\
& a \circ f(y)=a \circ e_{r}(y+\omega(y))=a(y+\omega(y)) / e_{r}^{\prime}(y+\omega(y))
\end{aligned}
$$


hence

$$
a_{n+1} / a_{n}=\frac{a(y+\omega(y))}{a(y)}\left(1+\omega^{\prime}(y)\right) .
$$

But by Lemma 6 and (15), $a(y+\omega(y)) / a(y)=1+0(\omega(y) / y)$ hence by (15), (16) and (20)

$$
a_{n+1} / a_{n}=1+0(1 / y)
$$

from which the convergence of (18) (and in fact its uniform convergence for $0<\xi \leqq x \leqq f(\xi)$ ) follows at once. The validity of (17) for this $b(x)$ and continuity of $b(x)$ follows from (18) and the uniformity of convergence. Finally (19) gives for $B(x)=\int^{x} b(t) d t$ and some constant $c$

$$
B \circ f(x)=B(x)+c \text {. }
$$

But clearly

$$
e_{r k-1}(x)<f_{k}(x)<e_{r k+1}(x)
$$

for every $k>1$ hence

$$
B \circ e_{r k-1}(x)<B(x)+k c<B \circ e_{r k+1}(x)
$$

and by (17)

$$
r k-1 \leqq k c \leqq r k+1
$$

for every $k>1$. Therefore $c=1$,

$$
B \circ f(x)=B(x)+1
$$

and $B(x)$ is indeed an Abel function of $f(x)$.

TheOREM 8. Suppose that $f(x)$ has a d.A.f. $b(x)$ such that

$$
\lim _{x \rightarrow \infty} a(x) / b(x)=r>0 .
$$

Then

$$
g(x)=e \circ f \circ e_{-1}(x)
$$

has a d.A.f. $b^{*}(x)$ such that

$$
\lim _{\infty \rightarrow \infty} a(x) / b^{*}(x)=r
$$

and $b^{*}(x)$ is uniquely determined by this condition.

Proof. Uniqueness of $b^{*}(x)$ follows as in Theorem 7. We show that $b^{*}(x)=(1+x)^{-1} \cdot b \circ e_{-1}(x)$ has the required property.

First, $b^{*}(x)$ is a d.A.f. of $g(x)$ by Lemma 2 with $\phi(x)=e_{-1}(x)=\log (1+x)$. Second, 
$\lim _{x \rightarrow \infty} a(x) / b^{*}(x)=\lim _{x \rightarrow \infty}(1+x) a(x) / b \circ e_{-1}(x)=\lim _{x \rightarrow \infty} r(1+x) a(x) / a \circ e_{-1}(x)$ by (17) and

$$
(1+x) a(x) / a \circ e_{-1}(x)=1
$$

by (3.2.)

COROLLARY. If $\omega(x)$ is as in Theorem 7 and $s$ is a non-negative integer then

$$
g(x)=e_{r+s}\left(e_{-s}(x)+\omega \circ e_{-\varepsilon}(x)\right)
$$

is regular with respect to iteration.

The corollary follows from Theorems 6, 7 and 8 .

Leмma 7. To every $L$-function $g(x)$ which tends to infinity there is a uniquely determined positive real number $\mu$ and non-negative integers $r, s$ such that for every $\delta, 0<\delta<\mu$,

$$
e_{r} \circ\left(e_{-s}(x)\right)^{\mu-\delta} \prec g(x) \prec e_{r} \circ\left(e_{-s}(x)\right)^{\mu+\delta} .
$$

This is one of Hardy's main results concerning the logarithmico-exponential scale and is proved in [4]. We shall say that $g(x)$ is of the type $(r, s, \mu)$ if it has the property (22).

Now suppose that $g(x)$ is an $L$-function of the type $(r, s, \mu)$ with $r \geqq s$. Then

$$
\begin{aligned}
& e_{r-s}\left(x^{\mu-\delta}\right)<e_{-s} \circ g \circ e_{s}(x)<e_{r-s}\left(x^{\mu+\delta}\right), \\
& e_{r-s}((\mu-\delta) x)<e_{-s-1} \circ g \circ e_{s+1}(x)<e_{r-s}((\mu+\delta) x), \\
& e_{r-s}(x+\log (\mu-\delta))<e_{-s-2} \circ g \circ e_{s+2}(x)<e_{r-8}(x+\log (\mu+\delta)) .
\end{aligned}
$$

Hence $\omega(x)$ in

$$
f(x)=e_{-s-2} \circ g \circ e_{s+2}(x)=e_{r-s}(x+\omega(x))
$$

is bounded. Since $\omega(x)$ is an $L$-function it is ultimately monotonic ([3], Theorem 13). Suppose now that $r>s$, then by the corollary of Theorem 8 $f(x)$ is regular with respect to iteration, and we have

Theorem 9. Every L-function $g(x)$ of the type $(r, s, \mu), r>s$ possesses a unique d.A.f. $b(x)$ characterized by the property

$$
\lim _{x \rightarrow \infty} a(x) / b(x)=r-s .
$$

If $g(x)$ is an $L$-function of the type $(r, r, \mu)$ then (23) gives

$$
f(x)=e_{-8-2} \circ g \circ e_{s+2}(x)=x+\omega(x)
$$

where $\omega(x)$ is bounded and ultimately monotonic. Hence formula (1.7) 
applies and $f(x)$ has a uniquely determined family of fractional iterates $f_{\sigma}(x)=x+\omega_{\sigma}(x)$ satisfying (4). By Theorem $5, f(x)$ is regular with respect to iteration and the regular iterates are given by

$$
g_{\sigma}(x)=e_{s+2} \circ f_{\sigma} \circ e_{--2}(x) .
$$

Thus we obtain a unique family of "best" iterates for every $L$-function of the type $(r, s, \mu), r \geqq s$.

If $r<s$ then we apply Theorem 9 to the inverse of $g(x)$ which satisfies a relation of the type (22) with $r>s$ ([4], p. 86). Hence we have proved:

THEOREM 10. Every L-function possesses a unique family of regular fractional iterates.

In conclusion we mention some unsolved problems.

1. Suppose that $f(x)$ is analytic on the positive real axis. Are the regular fractional iterates also analytic? The method of proof of Theorem 7 only gives the continuity of the first derivative and for instance it does not even allow us to decide whether the regular iterates of $e^{x}$ are analytic.

2. Is there a smallest extension $\boldsymbol{H}^{*}$ of $\boldsymbol{H}$ with the property that besides being closed under the rational operations and exp and log, it is also closed under regular fractional iteration? That is, any $f \in \boldsymbol{H}^{*}$ which tends to infinity, is regular with respect to iteration and its regular iterates also belong to $\boldsymbol{H}^{*}$.

3. If the system $\boldsymbol{H}^{*}$ exists, is it true that all members of $\boldsymbol{H}^{*}$ are ultimately monotonic and comparable with each other? Do these properties still hold if we require $H^{*}$ to be closed under differentiation?

For the ultimate acceptance of $a(x)$ as a new standard order of infinity it would evidently be desirable to have an affirmative answer to all these questions.

\section{References}

[1] Baker, I. N., Zusammensetzungen ganzer Funktionen, Math. Z., 69 (1958), 121-163.

[2] Bourbaki, N., Eléments de Mathématique, Book 4, Chapter 7 (Paris, 1951).

[3] Hardy, G. H., Orders of Infinity, Cambridge Tracts No. 12, 2-nd Edn. (Cambridge, 1824).

[4] Hardy, G. H., Properties of logarithmico-exponential functions, Proc. London Math. Soc. (2) 10 (1912), 54-90.

[5] Kneser, H.; Reelle analytische Losungen der Gleichung $\phi(\phi(x))=e^{a}$, J. f. reine angew. Math., 187 (1950), 56-67.

[6] Königs, G., Recherches sur les intégrales des certaines equations fonctionelles, Ann. Sci. Ecole Norm. Sup. (3) 1 (1884), Supplément, 3-41.

[7] Kuczma, M., On convex solutions of the functional equation $g[\alpha(x)]-g(x)=\phi(x)$, Publ. Math. Debrecen, 6 (1959) 40-47.

[8] Lévy, P., Fonctions à croissance régulière et itération d’ordre fractionnaire, Ann. Mat. Pura Appl. (4) 5 (1928), 269-298.

[9] Morris, K. W. and Szekeres, G., Tables of the logarithm of iteration of $e^{*}-1$. 
[10] Szekeres, G., Regular iteration of real and complex functions, Acta Math. 100 (1958), $203-258$.

[11] Szekeres, G., On a Theorem of P. Lévy, Publ. Math. Inst., Hungarian Acad. Sci., Ser A, $5(1960), 277-282$.

The University of Adelaide, South Australia. 